\title{
Large terahertz electric dipole of a single graphene quantum dot
}

\author{
Simon Messelot $\odot,{ }^{1}$ Elisa Riccardi $\odot,{ }^{1}$ Sylvain Massabeau, ${ }^{1}$ Federico Valmorra, ${ }^{1}$ Michael Rosticher, ${ }^{1}$ Kenji Watanabe $\odot,{ }^{2}$ \\ Takashi Taniguchi, ${ }^{3}$ Jérôme Tignon, ${ }^{1}$ Thomas Boulier,${ }^{1}$ Vincent Dauvois, ${ }^{4}$ Matthieu Delbecq $\odot,{ }^{1}$ Sukhdeep Dhillon, ${ }^{1}$ \\ Sébastien Balibar, ${ }^{1}$ Takis Kontos $\odot,{ }^{1}$ and Juliette Mangeney $\odot^{1, *}$ \\ ${ }^{1}$ Laboratoire de Physique de l'Ecole Normale Supérieure, ENS, Université PSL, CNRS, Sorbonne Université, \\ Université de Paris, F-75005 Paris, France \\ ${ }^{2}$ Research Center for Functional Materials, National Institute for Materials Science, Tsukuba, Ibaraki 305-0047, Japan \\ ${ }^{3}$ International Center for Materials Nanoarchitectonics, National Institute for Materials Science, Tsukuba, Ibaraki 305-0047, Japan \\ ${ }^{4}$ DEN/DPC/SECR/LRMO, CEA Saclay, F-91191 Gif sur Yvette Cedex, France
}

(Received 23 July 2021; accepted 4 January 2022; published 14 February 2022)

\begin{abstract}
Graphene has recently emerged as an important material for optoelectronic applications owing to its unique properties. Here, we show that graphene is also appealing for the development of $\mathrm{THz}$ quantum optics when used for quantum dots. In particular, we demonstrate a large electric dipole of length $d \approx 230 \mathrm{~nm}$ in a single graphene quantum dot, corresponding to an amplitude of $\approx 10 \mu \mathrm{eV}$ for the $\mathrm{THz}$ field. This estimate is done using transport measurements under coherent $\mathrm{THz}$ illumination and the well-established photon-assisted tunneling phenomenon for a spectral range larger than $100 \mathrm{GHz}$. Such large THz electric dipole for a quantum system that involves single-electron tunneling is suitable for future quantum optics experiments using graphene quantum dots strongly coupled to a $\mathrm{THz}$ cavity.
\end{abstract}

DOI: 10.1103/PhysRevResearch.4.L012018

\section{INTRODUCTION}

The use of light-matter interaction has enabled recent developments of nanotechnologies. New kinds a quantum devices such as quantum sensors and single-photon emitters rely on the interaction of photons with nanoscale structures. Graphene quantum dots (GQDs) are very attractive for quantum optoelectronic devices owing to their high flexibility in electronic states engineering through their size, shape, and edges [1-4]. Recently, room-temperature single optical photon emission with high purity and high brightness in the visible range has been demonstrated from GQDs of a few nanometers in diameter [5]. Furthermore, the demonstration of ultrasensitive detection of $\mathrm{THz}$ waves in large GQDs has recently been reported based on bolometric effects $[6,7]$ and photogating effects [8].

The response of GQDs to $\mathrm{THz}$ light, which could pave the way toward the development of novel quantum $\mathrm{THz}$ devices, has been elusive so far. Here, we demonstrate it using transport measurements under coherent $\mathrm{THz}$ illumination for a single GQD in the Coulomb blockade regime at low temperature. We observe specifically photon-assisted tunneling (PAT) over a broad spectral range $(>100 \mathrm{GHz})$ which allows us to extract a value of the $\mathrm{THz}$ electric dipole of length $d \approx 230 \mathrm{~nm}$, corresponding to an amplitude of $\approx 10 \mu \mathrm{eV}$ for

\footnotetext{
*Corresponding author: juliette.mangeney@phys.ens.fr
}

Published by the American Physical Society under the terms of the Creative Commons Attribution 4.0 International license. Further distribution of this work must maintain attribution to the author(s) and the published article's title, journal citation, and DOI. the $\mathrm{THz}$ field. These findings suggest a dipole extending over most of the active part of the device. Owing to their large dipole, GQDs coupled to a $\mathrm{THz}$ cavity are promising for ultrastrong coupling with only one electronic transition, paving the way toward probing nonclassical light and quantum-optical experiments.

\section{FABRICATION AND DARK TRANSPORT SPECTROSCOPY MEASUREMENTS}

We investigate a graphene quantum dot (GQD) as a single-electron transistor fabricated on an exfoliated graphene monolayer encapsulated by two hexagonal boron nitride $(\mathrm{hBN})$ layers [9]. E-beam lithography and reactive ion etching on the $\mathrm{hBN} /$ graphene heterostructure were used to define a large GQD of $120 \mathrm{~nm}$ diameter, connected to source and drain electrodes via two narrow constrictions $(40 \mathrm{~nm})$ that act as tunneling barriers, as presented in Fig. 1(a). The device also includes three lateral gate electrodes G1, G2, and G3. G1 acts on the chemical potential of the GQD while G2 and G3 set the constrictions to a nonconducting state $\left(V_{\mathrm{G} 2}=-3.45 \mathrm{~V}\right.$ and $V_{\mathrm{G} 3}=0 \mathrm{~V}$ ) $[10,11]$. As with the GQD, all the electrodes and constrictions are made of $\mathrm{hBN}$-encapsulated graphene, deposited on $\mathrm{SiO}_{2} / \mathrm{Si}$ substrate. The graphene electrodes are connected to gold electrodes with 1D contacts [12]. The device is placed in a dilution $4 \mathrm{He}-3 \mathrm{He}$ refrigerator with optical access. The sample temperature is $40 \mathrm{mK}$.

We initially perform dark transport spectroscopy measurements to explore the Coulomb blockade regime in the GQD. The differential conductance $\left(G_{\text {diff }}=\frac{d I}{d V_{\mathrm{DS}}}\right)$ as a function of $V_{\mathrm{G} 1}$ and $V_{\mathrm{DS}}$, displayed Fig. 1(b), exhibits the well-known Coulomb diamond shape showing the regime of sequential 
(a)

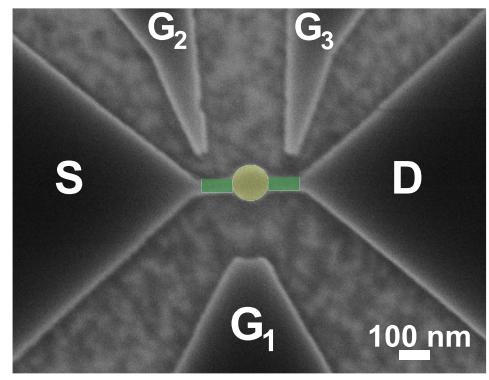

(b)

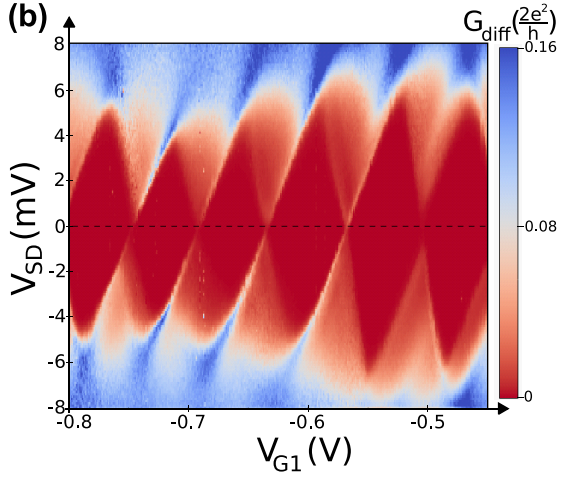

(c)

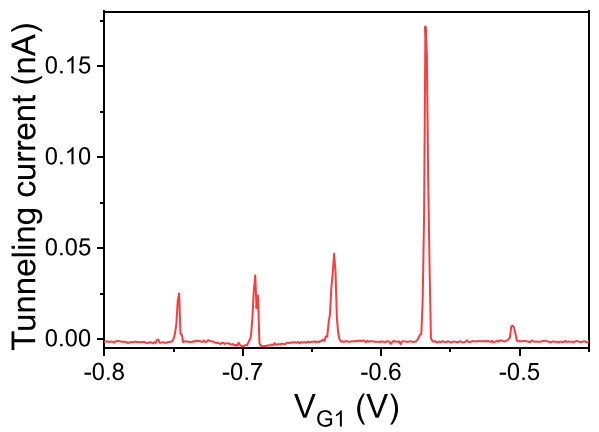

FIG. 1. (a) Scanning electron microscopy (SEM) image of the graphene section of the device, after etching of the hBN-graphene-hBN heterostructure. The picture shows the GQD (yellow) surrounded by the three side gates and linked to the source and drain electrodes. (b) Differential conductance $G_{\text {diff }}$ as a function of the source-drain voltage $V_{\mathrm{DS}}$ and of the plunger gate voltage $V_{\mathrm{G} 1}$, exhibiting Coulomb diamonds. Measurements are performed without THz illumination. (c) Dark DC current Coulomb peaks as a function of $V_{\mathrm{G} 1}$ at $V_{\mathrm{DS}}=250 \mu \mathrm{V}$.

tunneling where the transport is allowed only if the chemical potential of the source (or drain) crosses one empty level of the GQD [13]. Focusing on the region around $V_{\mathrm{G} 1}=$ $-0.6 \mathrm{~V}$, we extract from the diamond height the addition energy $E_{\text {add }} \sim 6 \mathrm{meV}$. The capacitive gate lever-arm factor is estimated through the diamond width to $\alpha_{G}=0.097$ (see Supplemental Material S3 for capacitive DC coupling description [14]).

The related Coulomb-blockade current peaks are observed in the tunneling current as a function of the plunger gate voltage $V_{\mathrm{G} 1}$ at low $V_{\mathrm{DS}}$ [Fig. 1(c)]. The differences between peak amplitudes, interpeak spacing, and also the observation of sequential tunneling of the electrons via the excited states of the GQD (lines parallel to the edges of the diamonds) are a signature that the graphene central island is small enough to behave as a quantum dot by opposition to a metallic island [4]. In this coupling regime, each Coulomb peak corresponds to a given GQD state, and only one electron is free to tunnel while the other electrons in the GQD are blocked by the GQD level spacing. The width of the Coulomb peaks is given by $\frac{\Gamma}{e \alpha_{G}}$ with $\Gamma$ the tunneling rate between the GQD and the source and drain leads. From Lorentzian fits, we extract Coulomb peak widths of about $2.6 \mathrm{mV}$ and we estimate $\Gamma \sim 0.25 \mathrm{meV}$. This value is in fair agreement with the estimation of $\Gamma \sim$ $0.31 \mathrm{meV}$ that is obtained from the $V_{\mathrm{DS}}$ scans of the differential conductance through the GQD [see Fig. 2(b) without illumination]. Indeed, the peak width is in this case given by $\frac{\Gamma}{e\left(1-\alpha_{S}\right)}$ with $\alpha_{S}$ the capacitive lever-arm factor of the source electrode.

\section{TRANSPORT UNDER THz ILLUMINATION}

We use a CW $\mathrm{THz}$ source consisting of GaAs Schottky diode frequency multipliers to investigate the response of the single GQD based device under coherent $\mathrm{THz}$ illumination. To focus the $\mathrm{THz}$ beam onto the GQD-based device, a pair of TPX lenses are placed in front of the refrigerator window. We use a $\times 9$ frequency multiplication chain to illuminate the GQD-based device with $\mathrm{THz}$ radiation at low frequencies, from 0.082 to $0.12 \mathrm{THz}$, and a $\times 27$ frequency multiplication chain for an illumination at high frequencies, from 0.24 to $0.38 \mathrm{THz}$. At low frequencies, since the photon energy $(\sim 0.45 \mathrm{meV})$ is of the order of the coupling rate to the electrodes $\Gamma \sim 0.3 \mathrm{meV}$, the interaction regime between the $\mathrm{THz}$ electric field and the GQD, which is probed, is considered as classical. By contrast, for high photon frequencies $>0.24$ $\mathrm{THz}$, as the photon energy $(\sim 1 \mathrm{meV})$ is larger than $\Gamma$, the quantum interaction regime is probed. In the following, we investigate successively the response of the GQD-based device under $\mathrm{THz}$ illumination in these two distinct interaction regimes.

\section{A. Transport under THz illumination: Classical regime}

We investigate the classical response of the single GQD in the Coulomb blockade regime at low temperature using transport measurements under $\mathrm{THz}$ illumination at $0.11 \mathrm{THz}$. Figure 2(a) shows the Coulomb stability diagrams without (left) and with $\mathrm{THz}$ irradiation (right). Under THz irradiation, we observe a nonzero conductance in regions of forbidden transport without illumination, i.e., inside the Coulomb diamonds. These results clearly highlight that excitation by $\mathrm{THz}$ waves modifies the tunneling process. Figure 2(b) shows the evolution of a differential conductance peak $\left(V_{\mathrm{G} 1}=\right.$ $-0.57 \mathrm{~V}$ ) for different incident $\mathrm{THz}$ intensity ranging from 0 to $49 \mu \mathrm{W} \mathrm{mm}{ }^{-2}$. Whereas the Coulomb blockade peak is sharp without illumination, with a width of $0.49 \mathrm{mV}$, with $\mathrm{THz}$ illumination, the peak broadens with increasing $\mathrm{THz}$ intensity. This evolution is also represented on the differential conductance map reported in Fig. 2(c). Indeed, the Coulombblockade peak spreads continuously as the $\mathrm{THz}$ intensity increases.

In a semiclassical picture, the broadening we observe is described by a modulation of the source-drain bias voltage by the incident $\mathrm{THz}$ electric field. In other words, the interaction of the incident $\mathrm{THz}$ electric field with the GQD-based device results in an $\mathrm{AC}$ voltage modulation of amplitude $V_{\mathrm{THz}}$ that is added to the source-drain bias, as depicted schematically in Fig. 2(b), right. The averaging of this modulation leads to a broadening of the conductance peaks. Interestingly, in this interaction mechanism and for $e V_{\mathrm{THz}}>\Gamma$, the full width at half maximum of the differential conductance peak under $\mathrm{THz}$ illumination is given by $\frac{\Gamma^{\prime}}{e} \approx 2 V_{\mathrm{THz}}$, allowing us to extract $V_{\mathrm{THz}}$ from measurements. To this aim, we fit the differential 

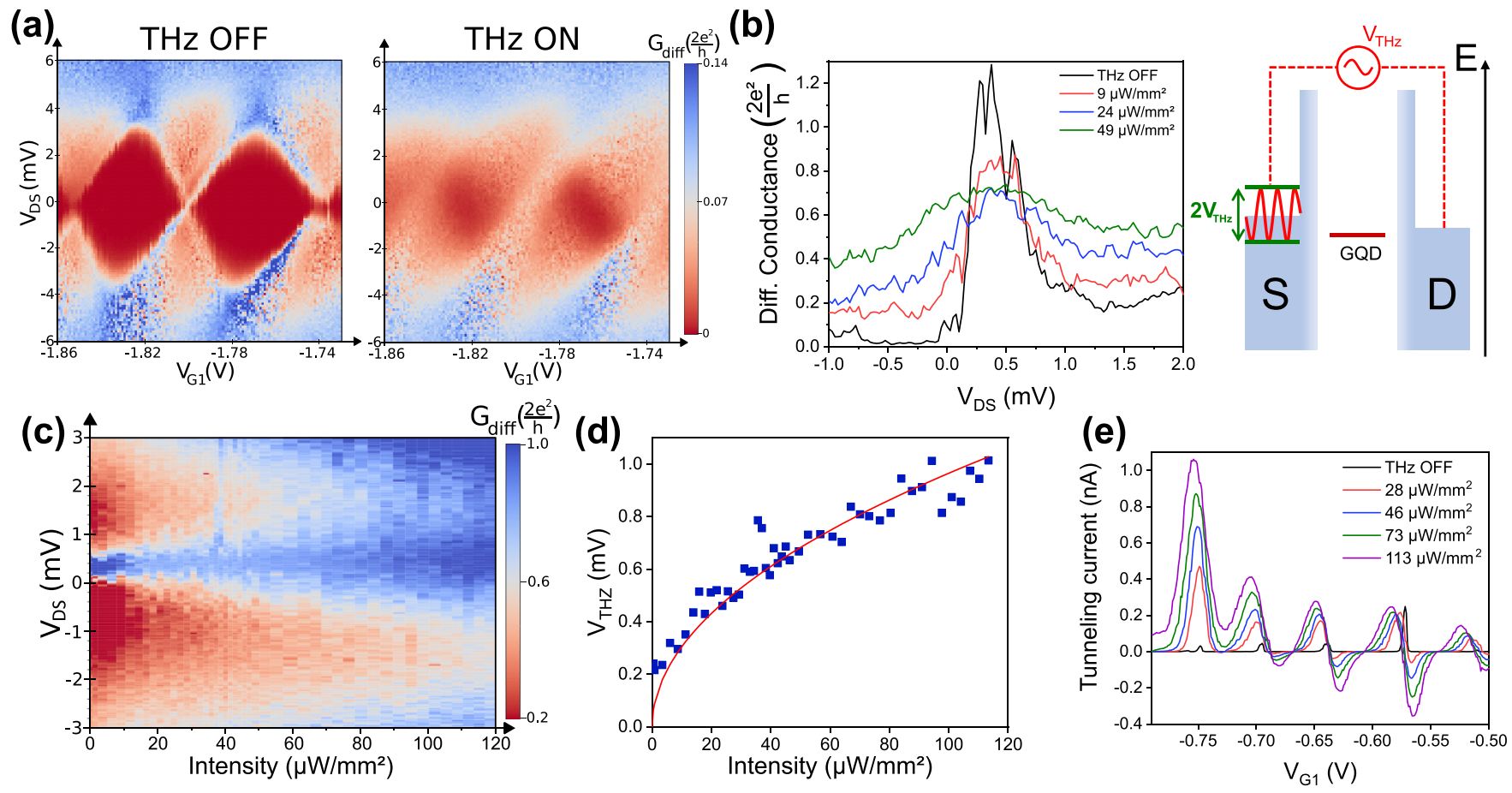

FIG. 2. (a) Differential conductance $G_{\mathrm{diff}}$ as a function of the source-drain voltage $V_{\mathrm{DS}}$ and of the plunger gate voltage $V_{\mathrm{G} 1}$ in dark (left) and with $\mathrm{THz}$ illumination at $0.11 \mathrm{THz}$ (right). (b) Left: Differential conductance as a function of $V_{\mathrm{DS}}$ and for $V_{\mathrm{G} 1}=-0.57 \mathrm{~V}$ without THz light (black curve) and for increasing incident THz intensity (color curves). Right: Schematic diagram of interaction scheme between the GQD and the THz waves. (c) Differential conductance map for $V_{\mathrm{DS}}$ scans as a function of the incident THz intensity $I_{\mathrm{THz}}$ at $V_{\mathrm{G} 1}=-0.57 \mathrm{~V}$. (d) $V_{\mathrm{THz}}$ extracted from measurements as a function of the incident $\mathrm{THz}$ intensity. (e) Tunneling current as a function of $V_{\mathrm{G} 1}$ for different incident $\mathrm{THz}$ intensities.

conductance peak with a Gaussian function: $A e^{-\frac{\left(V_{D S}-V_{0}\right)^{2}}{2 \sigma^{2}}}+$ $B\left(V_{\mathrm{DS}}-V_{1}\right)^{2}$, where $\sigma=0.43 \frac{\Gamma^{\prime}}{\rho}, A$ and $B$ are free parameters, and the second term is the background contribution (see details in Supplemental Material S3 [14]). The characteristic amplitude of this interaction $V_{\mathrm{THz}}$ extracted from measurements is reported in Fig. 2(d) as a function of the incident $\mathrm{THz}$ intensity, $I_{\mathrm{THz}}$. We observe that $V_{\mathrm{THz}}$ follows a square root dependence with $I_{\mathrm{THz}}$, which is consistent with a coherent interaction as $V_{\mathrm{THz}}$ scales like the electric field amplitude of the $\mathrm{THz}$ excitation.

Furthermore, we investigate the DC tunneling current through the GQD as a function of $V_{\mathrm{G} 1}$ for different incident $\mathrm{THz}$ intensities and for a positive $V_{\mathrm{DS}}$ bias. The $\mathrm{THz}$ radiation increases the current on the left side of the Coulomb blockade peak measured without illumination and induces a negative current on its right side. This change in sign of the current is attributed to an asymmetric $\mathrm{AC}$ coupling; i.e., the $\mathrm{AC}$ voltage drops across the two barriers are unequal $[15,16]$. When the ground-state level of the GQD is above the Fermi level of the leads, a nonzero net electron flows from the lead with the largest AC-coupled barrier to the other one, which is the pumped current. By changing $V_{\mathrm{G} 1}$, the level of the GQD is pulled below the Fermi energies of the reservoirs and the net electron flows now from the lead with the lowest AC-coupled barrier to the other one, which is an electron-photon pump $[15,17,18]$. This particular behavior highlights that the main response of our GQD-based device is nonthermal related. Also, it is a signature of the high nonlinearities exhibited by our GQD-based device, which is highly desirable for detection applications.

\section{B. Transport under THz illumination: Quantum regime}

We now extend the $\mathrm{THz}$ radiation frequency to the 0.24 $0.38 \mathrm{THz}$ band to explore the quantum interaction regime. The tunneling current $I_{\mathrm{DS}}$ as a function of $V_{\mathrm{G} 1}$ is reported in Fig. 3(a) for $V_{\mathrm{DS}}=100 \mu \mathrm{V}$ without (black line) and with (color lines) $\mathrm{THz}$ illumination. In addition to the Coulomb peaks observed without $\mathrm{THz}$ radiation, new satellite peaks appear on the right side of the principal Coulomb peaks under THz radiation. Furthermore, the satellite peak position is shown to shift in the direction of positive $V_{\mathrm{G} 1}$ as the frequency of the THz wave is increased. The energy spacing $e \alpha_{G} V_{\mathrm{G} 1}$ between the Coulomb peaks and the satellite peaks matches the photon energy of the THz wave, as shown in Fig. 3(b). This is a signature of photon-assisted tunneling [19,20]: the satellite peaks correspond to tunneling events through the GQD enabled by the absorption of a $\mathrm{THz}$ photon. We observe here a remarkably broadband quantized photoresponse extending from 0.24 to $0.38 \mathrm{THz}$, only limited by our $\mathrm{THz}$ source and the optical illumination setup (see detailed frequency domain investigations in Supplemental Material S1 [14]).

Photon-assisted tunneling in the $\mathrm{THz}$ range has been previously demonstrated in a few systems, i.e., semiconductor superlattices [21], resonant tunneling diodes [22], in single$\mathrm{C}_{60}$ molecules [23], in InAs quatum dots [24], and most recently carbon nanotubes [25-27], but remains elusive in 
(a)

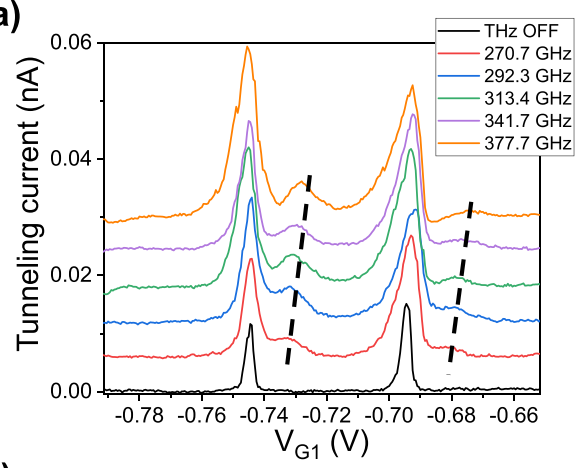

(b)

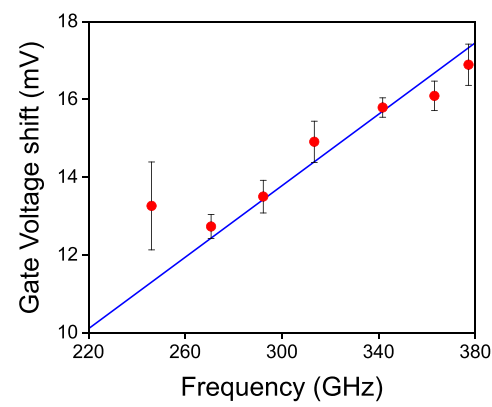

(c)

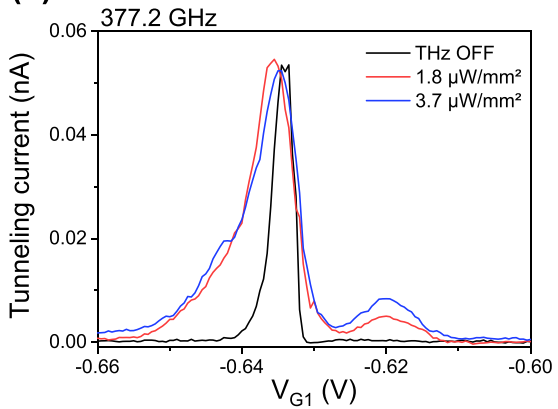

(d)

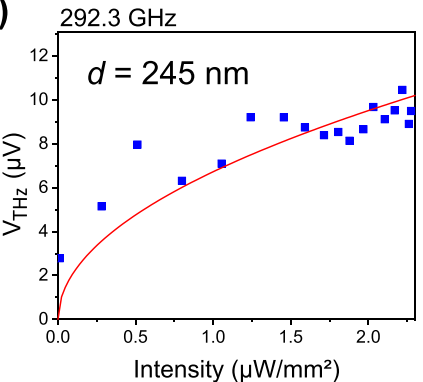

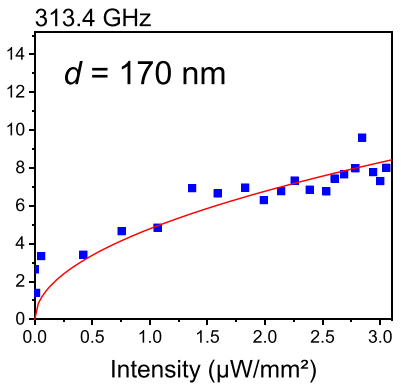

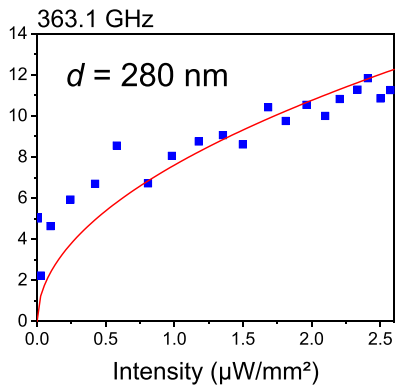

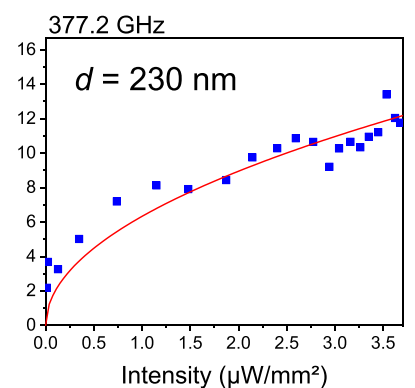

FIG. 3. (a) Source-drain current versus gate voltage $V_{\mathrm{G} 1}$ observed without (black curve) and with THz irradiation at frequencies $f=0.27$, $0.29,0.31,0.34$, and $0.38 \mathrm{THz}$ from bottom to top (color curves). The bias is $V_{\mathrm{DS}}=100 \mu \mathrm{V}$. The curves are offset by multiples of $0.006 \mathrm{pA}$ for clarity. The dashed lines show positions of satellite peaks due to photon-assisted tunneling process. (b) The energy spacing between the original Coulomb peaks and the satellite peaks as a function of the photon frequency of the $\mathrm{THz}$ wave. The blue line is a fit by a linear function. (c) The dark source-drain current versus $V_{\mathrm{G} 1}$ (black line) and the source-drain current under $\mathrm{THz}$ illumination with intensity of $1.8 \mu \mathrm{W}$ mm ${ }^{-2}$ (red line) and $3.7 \mu \mathrm{W} \mathrm{mm}^{-2}$ (blue line). (d) $\mathrm{THz}$ intensity dependence of the amplitude $V_{\mathrm{THz}}$ at frequencies $f=0.29,0.31,0.37$, and 0.36 THz. The red lines are square root fits from which are deduced the indicated dipole moment lengths $d$ (uncertainty $\sim 20 \%$ ).

GQDs. To gain further insight into these photoassisted tunneling processes, we study the dependence of the tunneling current on the incident $\mathrm{THz}$ intensity. Experimentally, we observe that the satellite current peak increases with the $\mathrm{THz}$ intensity, as observed in Fig. 3(c). We further perform the quantitative analysis of the satellite peak amplitude using the expression for the tunneling current $\langle J\rangle$ through a quantum dot under an oscillating bias voltage $V_{\mathrm{THz}}$ at frequency $\omega$ given by Jauho et al. [28]. The current is expressed as

$$
\begin{gathered}
\langle J\rangle\left(\mu_{L}, \mu_{R}, \omega, V_{\mathrm{THz}}, \epsilon_{d}\right)=-\frac{2 e}{\hbar} \frac{\Gamma^{L} \Gamma^{R}}{\Gamma^{L}+\Gamma^{R}} \\
\int \frac{d \epsilon}{2 \pi} \frac{\Gamma}{2}\left[f_{L}(\epsilon)-f_{R}(\epsilon)\right] \sum_{k=-\infty}^{+\infty} \frac{J_{k}^{2}\left(\frac{e V_{\mathrm{THz}}}{\hbar \omega}\right)}{\left(\epsilon-\epsilon_{d}-k \hbar \omega\right)^{2}+\left(\frac{\Gamma}{2}\right)^{2}}
\end{gathered}
$$

where $J_{k}$ is the $k$ th Bessel function of the first kind, $\mu_{L}$ and $\mu_{R}$ are the DC chemical potentials of source and drain electrodes, influencing $f_{L}(\epsilon)$ and $f_{R}(\epsilon)$ respectively, and $\epsilon_{d}=$ $\epsilon_{0}-\alpha_{G} e V_{\mathrm{G} 1}$ is the GQD energy level tuned during gate voltage scans. In Eq. (1), the peak function has a Lorentzian shape broadened by the Fermi distribution difference.

In this coupling scheme, the $\mathrm{THz}$ illumination modulates the chemical potentials of the electrodes at the frequency of the $\mathrm{THz}$ wave, with an amplitude $V_{\mathrm{THz}}$. It results in resonant tunneling at energy shifts equal to the photon energy that leads to the successive apparition of satellite peaks of amplitude $J_{k}^{2}\left(\frac{e V_{\mathrm{THz}}}{\hbar \omega}\right)$ centered at $e \alpha_{G} V_{\mathrm{G} 1}=\epsilon_{0}+k \hbar \omega$. The summation over $k$ accounts for the possible absorption of $k$ photons for the
PAT process. In the present case, we observe a single satellite peak corresponding to tunneling enabled by the absorption of one photon. We thereby limit the summation to $k=0$ and 1 in the above formula.

Using this expression, we fit the data of the evolution of the tunneling current with the incident $\mathrm{THz}$ intensity for several incident photon frequencies. This enables the evaluation of $V_{\mathrm{THz}}$ from the satellite peak amplitude (see Supplemental Material S4 for the exact integrated expression [14]). Figure 3(d) displays the extracted amplitude $V_{\mathrm{THz}}$ for 4 different $\mathrm{THz}$ frequencies and highlights its square root dependence on the incident $\mathrm{THz}$ intensity (red line). Note that from the usual PAT theory [28], a second satellite peak, symmetric to the one we observe, would be expected on the left side of the Coulomb blockade peak. Such symmetric peak is not present in our measurements, similarly to previous works [29-31]. We attribute the absence of this low $V_{\mathrm{G} 1}$ satellite peak to the asymmetric coupling of the $\mathrm{THz}$ wave to the source and drain electrodes in a regime where the bias $(100 \mu V)$ is larger than $V_{\mathrm{THz}}$ [32]. This observation is fully consistent with the change in sign of the current measured in the low-frequency regime.

\section{DISCUSSION}

The amplitude $V_{\mathrm{THz}}$ characterizes the interaction strength of the GQD-based device with the incident $\mathrm{THz}$ wave. As a relevant figure, the electric dipole moment length $d$ of the GQD is deduced from the relation $V_{\mathrm{THz}}=\vec{E}_{\mathrm{THz}} \cdot \vec{d}$. Since in our experiment, the electric field is polarized along the source-drain axis, this equation is reduced to a simple product. 
Quantitatively, we extract the electric field amplitude from the $\mathrm{THz}$ intensity (see details in Supplemental Material S2 [14]) and calculate the electric dipole moment length of the GQD $d$. The estimation of $d$ is reported in Fig. 3(d) for frequencies $f=0.29,0.31,0.37$, and $0.36 \mathrm{THz}$. We find an average electric dipole moment length $d \approx 230 \mathrm{~nm}(\sim 20 \%$ uncertainty), which is in the range of the distance between source and drain electrodes of $320 \mathrm{~nm}$ in the GQD-based device. This suggests that it is mainly between the two electrodes that the dipole is built. Our estimate of $d$ is fully consistent with an electric dipole that involves electronic transitions between an orbital of the reservoir electrons (source or drain) and an orbital of the graphene quantum dot, for which the spatial extension is expected between the edges of the reservoirs, where the reservoir electron orbitals have significant overlap with the graphene quantum dot orbitals. This matching between the electric dipole moment length $d$ deduced from measurements and the source-drain distance strongly supports the validity of our analysis.

\section{CONCLUSION}

As a conclusion, using low-temperature transport measurements under coherent $\mathrm{THz}$ illumination we have investigated the interaction between a single GQD and a THz electric field in the classical and quantum regime. We have demonstrated the photon-assisted tunneling phenomenon when the single GQD is illuminated by $\mathrm{THz}$ radiation in the broad $0.24-0.38$ $\mathrm{THz}$ band. From the experimental and theoretical study of the satellite photon-assisted current peaks, we have shown a large dipole for the electronic excitation with $d \approx 230 \mathrm{~nm}$. As a perspective toward strong resonant $\mathrm{THz}$ light-matter coupling via a cavity, we can estimate the coupling strength defined by $g \approx e d E_{\text {vac }}$ with $E_{\text {vac }}=\sqrt{\hbar \omega_{0} /\left(2 \varepsilon_{0} V_{\text {cav }}\right)}$ the vacuum field in the cavity and $V_{\text {cav }}$ the mode volume in the cavity. On the basis of our results and assuming confining the electric field into nanoscale volumes, of the order of $V_{\text {cav }} \sim 10^{-7} \lambda^{3}$, as reported by Gupta et al. at $0.6 \mathrm{THz}$ [33], we expect a reduced coupling constant $g / \omega_{0}>0.1$, indicating the possibility to reach the ultrastrong-coupling regime. For nanogap hybrid LC cavity with $V_{\text {cav }} \sim 6 \times 10^{-10} \lambda^{3}$ at $0.3 \mathrm{THz}$ recently reported by Keller et al. [34], the reduced coupling constant can even reach the deep strong-coupling regime $g / \omega_{0} \sim 1$. Remarkably, our system involves only one electronic transition (a single-electron transition) and not an ensemble as reported in systems put forward to reach the ultrastrong-coupling regime in the $\mathrm{THz}$ spectral range such as intersubband transitions and the cyclotron orbits. In addition, GQDs could be superior in terms of scalability with respect to recently developed carbon nanotube based platforms [35], as they can be patterned on a nanometer scale using a top-down approach with present lithography technology. Thus, owing to its large dipole $(\sim 230 \mathrm{~nm}), \mathrm{GQD}$ paves the way to study the ultrastrong THz light-matter coupling physics at the single-electron level, making it a promising platform for quantum technologies.

\section{ACKNOWLEDGMENTS}

The authors thank Pascal Morfin for his help with cryogenics. This project has received funding from the European Research Council (ERC) under the European Union's Horizon 2020 research and innovation program (Grant Agreement No. 820133). This research is supported by a public grant overseen by the French National Research Agency (ANR) as grants STEM2D (ANR-19-CE24-0015).
[1] M. Zarenia, A. Chaves, G. A. Farias, and F. M. Peeters, Energy levels of triangular and hexagonal graphene quantum dots: A comparative study between the tight-binding and Dirac equation approach, Phys. Rev. B 84, 245403 (2011).

[2] A. D. Güçlü, P. Potasz, and P. Hawrylak, Excitonic absorption in gate-controlled graphene quantum dots, Phys. Rev. B 82, 155445 (2010).

[3] C. Mansilla Wettstein, F. P. Bonafé, M. B. Oviedo, and C. G. Sánchez, Optical properties of graphene nanoflakes: Shape matters, J. Chem. Phys. 144, 224305 (2016).

[4] L. A. Ponomarenko, F. Schedin, M. I. Katsnelson, R. Yang, E. W. Hill, K. S. Novoselov, and A. K. Geim, Chaotic Dirac billiard in graphene quantum dots, Science 320, 356 (2008).

[5] S. Zhao et al., Single photon emission from graphene quantum dots at room temperature, Nat. Commun. 9, 3470 (2018).

[6] A. El Fatimy, R. L. Myers-Ward, A. K. Boyd, K. M. Daniels, D. K. Gaskill, and P. Barbara, Epitaxial graphene quantum dots for high-performance terahertz bolometers, Nat. Nanotechnol. 11, 335 (2016).

[7] A. E. Fatimy, A. Nath, B. D. Kong, A. K. Boyd, R. L. MyersWard, K. M. Daniels, M. M. Jadidi, T. E. Murphy, D. K. Gaskill, and P. Barbara, Ultra-broadband photodetectors based on epitaxial graphene quantum dots, Nanophotonics 7, 735 (2018).
[8] E. Riccardi, S. Massabeau, F. Valmorra, S. Messelot, M Rosticher, J. Tignon, K. Watanabe, T. Taniguchi, M. Delbecq, S. Dhillon et al., Ultrasensitive photoresponse of graphene quantum dots in the Coulomb blockade regime to $\mathrm{THz}$ radiation, Nano Lett. 20, 5408 (2020).

[9] L. Banszerus, M. Schmitz, S. Engels, J. Dauber, M. Oellers, F. Haupt, K. Watanabe, T. Taniguchi, B. Beschoten, and C. Stampfer, Ultrahigh-mobility graphene devices from chemical vapor deposition on reusable copper, Sci. Adv. 1, e1500222 (2015).

[10] C. Stampfer, J. Güttinger, F. Molitor, D. Graf, T. Ihn, and K. Ensslin, Tunable Coulomb blockade in nanostructured graphene, Appl. Phys. Lett. 92, 012102 (2008).

[11] J. Güttinger, F. Molitor, C. Stampfer, S. Schnez, A. Jacobsen, J. Droscher, T. Ihn, and K. Ensslin, Transport through graphene quantum dots, Rep. Prog. Phys. 75, 126502 (2012).

[12] L. Wang, I. Meric, P. Y. Huang, Q. Gao, Y. Gao, H. Tran, T. Taniguchi, K. Watanabe, L. M. Campos, D. A. Muller, J. Guo, P. Kim, J. Hone, K. L. Shepard, and C. R. Dean, One-dimensional electrical contact to a two-dimensional material, Science $\mathbf{3 4 2}$ 614 (2013)

[13] S. Schnez, F. Molitor, C. Stampfer, J. Guttinger, I. Shorubalko, T. Ihn, and K. Ensslin, Observation of excited states in a graphene quantum dot, Appl. Phys. Lett. 94, 012107 (2009). 
[14] See Supplemental Material at http://link.aps.org/supplemental/ 10.1103/PhysRevResearch.4.L012018 for a detailed discussion of the frequency dependence of the photoresponse, $\mathrm{THz}$ intensity calibration measurements, useful expressions of the current and the differential conductance in the Coulomb blockade regime, and the derivation of the tunneling current expression under illumination, which includes Refs. [36-38].

[15] L. P. Kouwenhoven, S. Jauhar, K. McCormick, D. Dixon, P. L. McEuen, Y. V. Nazarov, N. C. van der Vaart, and C. T. Foxon, Photon-assisted tunneling through a quantum dot, Phys. Rev. B 50, 2019 (1994).

[16] W. G. Van der Wiel, T. Oosterkamp, S. de Franceschi, C. Harmans, and L. Kouwenhoven, Photon-assisted tunneling in quantum dots, in Strongly Correlated Fermions and Bosons in Low-Dimensional Disordered Systems (Springer, Dordrecht, 2002), pp. 43-68.

[17] R. H. Blick, R. J. Haug, D. W. van der Weide, K. von Klitzing, and K. Eberl, Photon-assisted tunneling through a quantum dot at high microwave frequencies, Appl. Phys. Lett. 67, 3924 (1995).

[18] T. H. Oosterkamp, L. P. Kouwenhoven, A. E. A. Koolen, N. C. van der Vaart, and C. J. P. M. Harmans, Photon induced resonances in the current through a quantum dot with zero-dimensional states, Phys. Scr. T69, 98 (1997).

[19] L. P. Kouwenhoven, S. Jauhar, J. Orenstein, P. L. McEuen, Y. Nagamune, J. Motohisa, and H. Sakaki, Observation of PhotonAssisted Tunneling through a Quantum Dot, Phys. Rev. Lett. 73, 3443 (1994)

[20] T. H. Oosterkamp, L. P. Kouwenhoven, A. E. A. Koolen, N. C. van der Vaart, and C. J. P. M. Harmans, Photon Sidebands of the Ground State and First Excited State of a Quantum Dot, Phys. Rev. Lett. 78, 1536 (1997).

[21] S. Zeuner, S. Allen, K. Maranowski, and A. Gossard, Photon-assisted tunneling in GaAs/AlGaAs superlattices up to room temperature, Appl. Phys. Lett. 69, 2689 (1996).

[22] H. Drexler, J. Scott, S. Allen, K. Campman, and A. Gossard, Photon-assisted tunneling in a resonant tunneling diode: Stimulated emission and absorption in the THz range, Appl. Phys. Lett. 67, 2816 (1995).

[23] K. Yoshida, K. Shibata, and K. Hirakawa, Terahertz Field Enhancement and Photon-Assisted Tunneling in Single-Molecule Transistors, Phys. Rev. Lett. 115, 138302 (2015).

[24] K. Shibata, A. Umeno, K. M. Cha, and K. Hirakawa, PhotonAssisted Tunneling through Self-Assembled InAs Quantum Dots in the Terahertz Frequency Range, Phys. Rev. Lett. 109, 077401 (2012).
[25] C. Meyer, J. M. Elzerman, and L. P. Kouwenhoven, Photonassisted tunneling in a carbon nanotube quantum dot, Nano Lett. 7, 295 (2007)

[26] T. Tsurugaya, K. Yoshida, F. Yajima, M. Shimizu, Y. Homma, and K. Hirakawa, Terahertz spectroscopy of individual carbon nanotube quantum dots, Nano Lett. 19, 242 (2019).

[27] M. Rinzan, G. Jenkins, H. D. Drew, S. Shafranjuk, and P. Barbara, Carbon nanotube quantum dots as highly sensitive terahertz-cooled spectrometers, Nano Lett. 12, 3097 (2012).

[28] A.-P. Jauho, N. S. Wingreen, and Y. Meir, Time-dependent transport in interacting and noninteracting resonant-tunneling systems, Phys. Rev. B 50, 5528 (1994).

[29] T. Fuse, Y. Kawano, T. Yamaguchi, Y. Aoyagi, and K. Ishibashi, Quantum response of carbon nanotube quantum dots to terahertz wave irradiation, Nanotechnology 18, 044001 (2007).

[30] Y. Kawano, T. Fuse, S. Toyokawa, T. Uchida, and K. Ishibashi, Terahertz photon-assisted tunneling in carbon nanotube quantum dots, J. Appl. Phys. 103, 034307 (2008).

[31] K. Ishibashi, S. Moriyama, T. Fuse, Y. Kawano, S. Toyokawa, and T. Yamaguchi, Artificial atom and quantum terahertz response in carbon nanotube quantum dots, J. Phys.: Condens. Matter 20, 454203 (2008).

[32] Q.-f. Sun and T.-h. Lin, Influence of microwave fields on the electron tunneling through a quantum dot, Phys. Rev. B 56, 3591 (1997).

[33] M. Gupta and R. Singh, Terahertz sensing with optimized $Q / V_{\text {eff }}$ metasurface cavities, Adv. Optical Mater. 8, 1902025 (2020).

[34] J. Keller, G. Scalari, S. Cibella, C. Maissen, F. Appugliese, E. Giovine, R. Leoni, B. Beck, and J. Faist, Few-electron ultrastrong light-matter coupling at $300 \mathrm{GHz}$ with nanogap hybrid LC microcavities, Nano Lett. 17, 7410 (2017).

[35] F. Valmorra, K. Yoshida, L. C. Contamin, S. Messelot, S. Massabeau, M. R. Delbecq, M. C. Dartiailh, M. M. Desjardins, T. Cubaynes, Z. Leghtas, K. Hirakawa, J. Tignon, S. Dhillon, S. Balibar, J. Mangeney, A. Cottet, and T. Kontos, Vacuum-fieldinduced $\mathrm{THz}$ transport gap in a carbon nanotube quantum dot, Nat. Commun. 12, 5490 (2021).

[36] D. Grischkowsky, S. Keiding, M. Van Exter, and C. Fattinger, Far-infrared time-domain spectroscopy with terahertz beams of dielectrics and semiconductors, J. Opt. Soc. Am. B 7, 2006 (1990).

[37] M. Hofheinz, X. Jehl, M. Sanquer, G. Molas, M. Vinet, and S. Deleonibus, Capacitance enhancement in Coulomb blockade tunnel barriers, Phys. Rev. B 75, 235301 (2007).

[38] Y. Meir and N. S. Wingreen, Landauer Formula for the Current through an Interacting Electron Region, Phys. Rev. Lett. 68, 2512 (1992). 